\title{
Moluscos límnicos de uma área do Alto Araguaia (Brasil)*
}

\author{
Limnic molluscs from Alto Araguaia area (Brazil)
}

\begin{abstract}
Horácio Manuel Santana Teles ${ }^{\star *}$, Renata Piva Almeida Leite***, Francisco Luiz Rodrigues**
TELES, H.M.S. et al. Moluscos límnicos de uma área do Alto Araguaia (Brasil). Rev. Saúde públ., S. Paulo, 25: 179-83, 1991. São mostrados os resultados do levantamento dos moluscos de água doce de 5 municípios da área de influência de uma usina hidrelétrica em estudo, formada pelas redondezas da Cachoeira Couto Magalhães no Rio Araguaia. Foram examinados 624 moluscos procedentes de amostras coletadas em 20 coleções hídricas. O exame revelou a ocorrência de 8 gastrópodos pulmonados e um prosobrânquio, além de um bivalvo esferideo. Devido ao envolvimento com a saúde pública, merece destaque o encontro de Biomphalaria straminea e Lymnaea columella, respectivamente hospedeiros intermediários de Schistosoma mansoni e Fasciola hepatica.
\end{abstract}

Descritores: Moluscos. Vetores de doenças. Esquistossomose mansônica. Fasciolíase.

\section{Introdução}

No intuito de reunir conhecimentos para a análise mais apropriada sobre a receptividade às doenças transmitidas por moluscos na área de influência da implantação da Usina Hidrelétrica Couto de Magalhães (UHE), no Rio Araguaia, realizou-se levantamento da malacofauna de 5 municípios: Alto Araguaia e Alto Taquari (Estado de Mato Grosso - MT), Mineiros, Portelândia e Santa Rita do Araguaia (Estado de Goiás - GO).

É oportuno salientar a validade das pesquisas nas áreas de construção e implantação de hidrelétricas, porque estes empreendimentos costumam atrair pessoas de vários pontos do país, eventualmente albergando parasitos que são transmitidos com a participação de determinadas espécies dos moluscos de água doce, como o Schistosoma mansoni. Em conseqüência da entrada de portadores humanos nas áreas de influência dos projetos, no caso das condições serem favoráveis, isto é, pela presença de hospedeiros intermediários adequados e deficiência do saneamen-

* Patrocinado pelas Centrais Elétricas do Norte do Brasil S.A. (ELETRONORTE) e executados pelas equipes da Superintendência de Campanhas de Saúde Pública (SUCAM) e do Consórcio Nacional de Engenheiros Consultores S.A. (CNEC).

** Superintendencia de Controle de Endemias (SUCEN) São Paulo, SP - Brasil

*** Consórcio Nacional de Engenheiros Consultores (CNEC) - São Paulo, SP - Brasil.

Separatas/Reprints: H.M.S. Teles - Rua Paula Souza, 166 - 01027 - São Paulo, SP - Brasil.

Publicação financiada pela FAPESP. Processo Medicina 90/4602-1 to ambiental, coloca-se em risco a mudança do panorama epidemiológico das áreas indenes, mediante a possibilidade da formação de focos de doenças até então desconhecidas. No caso da esquistossomose, a questão foi discutida por Camargo $^{1}$ e Saif e Gaber ${ }^{9}$.

Como os conhecimentos sobre a fauna de moluscos predominantes nas áreas afetadas pela construção e funcionamento das hidrelétricas são fundamentais para o delineamento da situação sanitária, apresentam-se os resultados do levantamento efetuado em maio e julho de 1989 que, associados a outras informações de caráter epidemiológico, permitem traçar um perfil preliminar das condições existentes para a instalação de doenças relacionadas com moluscos.

\section{Material e Método}

Foram examinados 624 exemplares de moluscos de 20 amostras coletadas mediante a pesquisa de 26 ambientes límnicos distintos. O material colhido foi tratado conforme o preconizado por Paraense?.

Para a identificação das espécies foram considerados os detalhes morfológicos das conchas e do sistema genital.

\section{Resultados}

Os exames de laboratório consignaram as seguintes espécies: Drepanotrema anatinum (d'Orbigny, 1835), D. lucidum (Pfeiffer, 1839), D. depressissimum (Moricand, 1839), Biomphalaria 
straminea (Dunker, 1848), B. schrammi (Crosse, 1849), Physa marmorata (Guilding, 1828), Lymnaea columella (Say, 1817), Pomacea Perry, 1810 e Eupera Bourguignat, 1854. Logo, foram reconhecidos 9 gastrópodos, sendo 8 pulmonados e um prosobrânquio, e um bivalvo. Os dois últimos grupos (Pomacea e Eupera) foram classificados genericamente porque os estudos taxionômicos ainda são insuficientes ao perfeito enquadramento das espécies.

$\mathrm{Na}$ Tabela e no Anexo aparecem as espécies encontradas por município, locais de coleta e o número de exemplares examinados por espécie, mostrando que as ocorrências de moluscos aconteceram em $20(\mathbf{7 6 , 9 2 \% )}$ das coleções hídricas perqueridas, dentre as quais, $7(35 \%)$ comportavam espécies em sintopia.

Ainda na Tabela e no Anexo, Drepanotre$m a$ evidencia ser o gênero mais freqüente na área, seguido por Biomphalaria, Pomacea, Eupera, Physa e Lymnaea. Os representantes da família Planorbidae foram mais abundantes, colonizando $16(61,53 \%)$ criadouros, e correspondendo com $67,14 \%$ do material estudado.

A Figura representa a área de influência da UHE, a localização dos pontos de coleta e as espécies encontradas. Apesar da existência desses pontos nos municípios de Alto Taquari e Portelândia, não foi verificada a ocorrência de moluscos.

Tabela. Exemplares e espécies dos moluscos limnicos coletados na área de influência da Usina Hidrelétrica Couto de Magalhães, em maio e julho de 1989.

\begin{tabular}{lrrrrrrrrrr}
\hline Municípios & \multicolumn{10}{c}{ Espécies } \\
\cline { 2 - 10 } & Da & Dl & Dd & Bs & Bsc & Pm & Lc & P & E \\
\hline Alto Araguaia & 0 & 50 & 59 & 0 & 0 & 0 & 0 & 35 & 0 \\
Alto Taquari & 0 & 0 & 0 & 0 & 0 & 0 & 0 & 0 & 0 \\
Mineiros & 33 & 0 & 2 & 116 & 87 & 33 & 3 & 22 & 43 \\
Portelândia & 0 & 0 & 0 & 0 & 0 & 0 & 0 & 0 & 0 \\
Santa Rita do & & & & & & & & & \\
Araguaia & 0 & 70 & 2 & 0 & 0 & 0 & 0 & 68 & 1 \\
\hline Total & 33 & 120 & 63 & 116 & 87 & 33 & 3 & 125 & 44
\end{tabular}

$\mathrm{Da}=D$. anatinum, $\mathrm{DI}=D$. lucidum; $\mathrm{Dd}=D$. depressissimum, Bs $=B$. straminea; Bsc $=B$. schrammi, $\mathrm{Pm}=$ $P$. marmorata $\mathrm{LC}=\mathrm{L}$. columella; $\mathrm{P}=$ Pomacea; $\mathrm{E}=$ Eupera.

\section{Comentários}

Paraense $^{8}$ já havia constatado a ocorrência de $D$. lucidum, $D$. depressissimum e $B$. schram$m i$ no município de Alto Araguaia, sendo estes os únicos conhecimentos sobre a fauna dos moluscos de água doce presentes na área de Couto Magalhães, publicados até o momento. Com os resultados de agora fica conferida a existência de mais espécies, inclusive de interesse sanitá- rio, como B. straminea e $L$. columella, bem como pôde-se observar melhor adaptação dos planorbídeos (Drepanotrema e Biomphalaria) à ecologia local.

O encontro de $B$. straminea foi particularmente importante, dado o envolvimento da espécie na transmissão da esquistossomose em várias localidades do Brasil, mormente da região nordeste. Embora $B$. straminea não responda experimentalmente como o transmissor mais suscetível de $S$. mansoni, na natureza parece conjugar fatores biológicos e ecológicos capazes de manter prevalências elevadas da endemia. Paraense $^{6}$ postula que as dificuldades das relaçőes hospedeiro intermediário/parasito são suficientemente compensadas pelas grandes densidades populacionais manifestadas pelo caramujo e pela capacidade de ocupação dos mais variados tipos de habitats e climas. Estas características garantem a ampla distribuição geográfica de $B$. straminea. Com a aparente abundância de $B$. straminea nos criadouros de Mineiros (quase $20 \%$ do material examinado), fica consubstanciada a observação do autor citado. É bem provável que este importante transmissor da esquistossomose, no futuro, venha a ser descoberto em mais localidades de Mineiros e nos municípios vizinhos.

Do ponto de vista epidemiológico, a proximidade dos criadouros de $B$. straminea, com as residências do homem, também merece destaque. A contaminação dos ambientes hídricos peridomiciliares por esgotos foi constante, trazendo maiores facilidades para o contágio por $S$. mansoni, uma vez que Doumenge e col. ${ }^{4}$ registraram a existência de casos de esquistossomose no município. No sentido de avaliar a endemia na atualidade, seria de bom alvitre a realização de um inquérito parasitológico em Mineiros, junto à periferia da cidade. Doumenge e col. ${ }^{4}$ também mostram a presença de portadores de $S$. mansoni no município de Alto Araguaia. Estes casos devem ser importados porque não foram detectados hospedeiros intermediários no município.

A segunda espécie de molusco dotada de interesse para a saúde pública presente em Mineiros, é $L$. columella. Esta espécie de pulmonado é hospedeira de Fasciola hepatica. Aparecendo em um único criadouro, limita sobremaneira as possibilidades da introdução e propagação do parasito, apesar de Dacal e col. ${ }^{3}$ apregoarem a razoável suscetibilidade do limneídeo e a existência de casos de fasciolose no centro-oeste brasileiro. Com a restrita distribuição, é remota a disseminação de $F$. hepatica na área de influência da UHE.

Para concluir, de uma maneira geral, ex- 


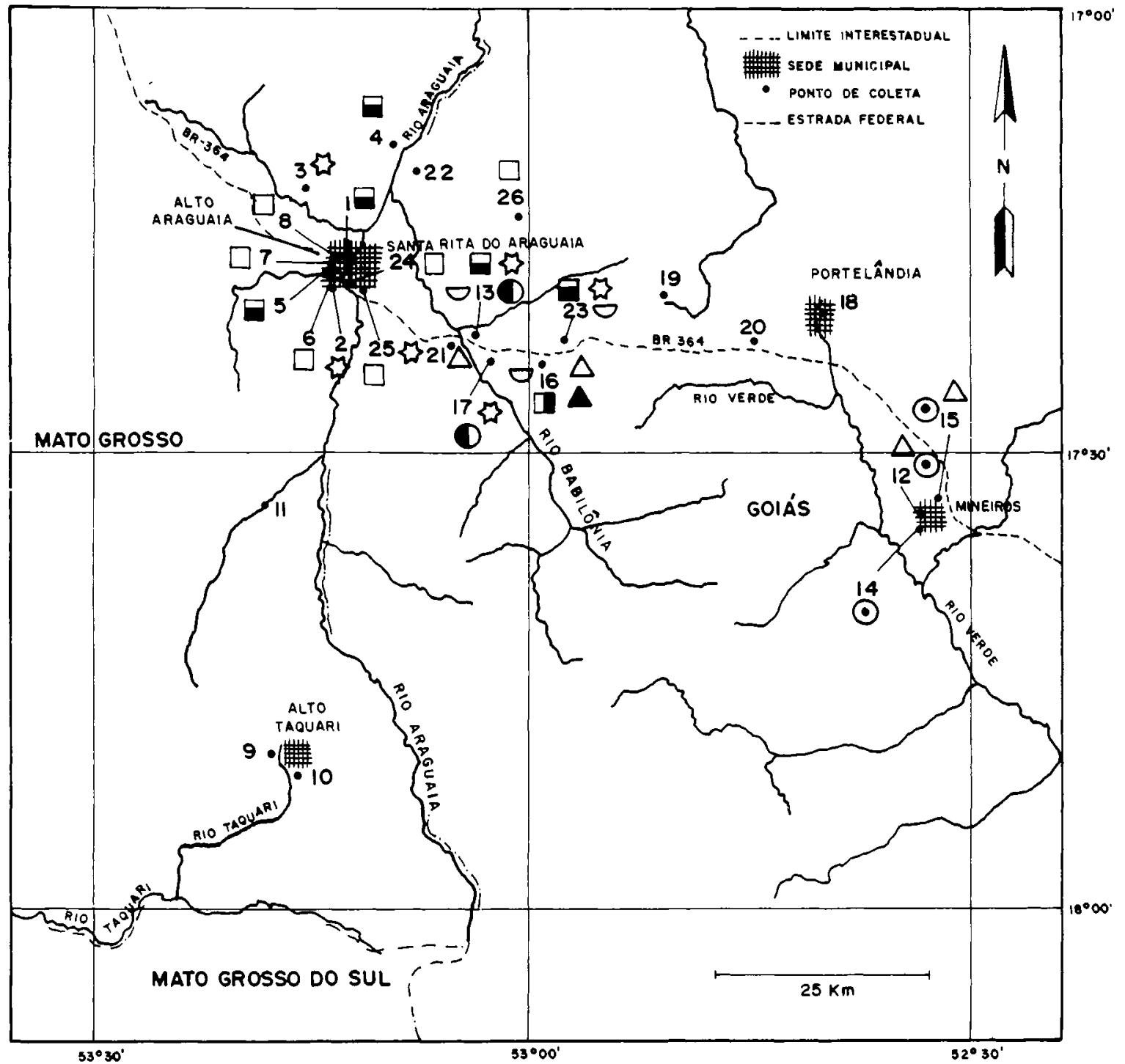

Figura - Distribuição dos criadouros e espécies encontradas na área de influência da Usina Hidrelétrica Couto de Magalhães $(\square=D$. lucidum; $\square=D$. depressissimum; $\square=D$. anatinum; $\odot=$ B. straminea; $D=B$. schrammi; $\Delta=$ P. marmorata; $\boldsymbol{\Delta}=$ L. columella; = Pomacea; $\square=$ Eupera ).

cluindo-se a situação instalada em Mineiros que já é preocupante, é pouco provável que as obras e o funcionamento da hidrelétrica tragam, no bojo dos impactos ambientais causados, motivo de preocupação no que tange à hipótese da introdução da esquistossomose ou fasciolose. A afirmação leva em conta a inexistência de espécies hospedeiras intermediárias dessas endemias na maior parte da área investigada. Todavia é necessário consentir a introdução posterior de moluscos, pois não são raros os casos de dispersão em projetos de repovoamento, cultura de peixes e plantas ornamentais para aquários.
Assim é prudente o acompanhamento periódico da fauna instalada na área de influência da UHE. A vigilância constante sobre a fauna de moluscos é aconselhável porque a região centrooeste, onde será instalado o projeto, é receptiva à formação de focos de $S$. mansoni, como atestam Cunha $\mathrm{Neto}^{2}$, em Goiânia (GO), Magalhães ${ }^{5}$, em Brasília (DF) e Camargo ${ }^{1}$, em Cáceres (MT).

Paralelamente, a freqüência tão amiúde da coexistência interespecifica (sintopia) na área, oferece condições excelentes ao desenvolvimento de estudos dos efeitos competitivos entre mo- 
luscos, detectando na natureza espécies que eventualmente podem atuar, reduzindo, eliminando ou deslocando as populações dos hospedeiros intermediários de $S$. mansoni e $F$. hepatica.

\section{Agradecimentos}

Ao Dr. Ernane G. Fernandes da Motta, Diretor Geral do DECEN/SUCAM, aos diretores e equipes das Diretorias Regionais da Sucam de Goiás e Mato Grosso, pelo apoio e incentivo, sem o qual o trabalho seria muito dificultado.

TELES, H.M.S. et al. [Limnic molluses from Alto Araguaia area (Brazil)]. Rev. Saúde públ., S. Paulo, 25: 179-83, 1991. An analysis was carried out to determine the natural population of freshwater molluscs from 5 municipalities within the area of influence of the Couto Magalhães hidroeletric project. These municipalities cover a large area of the State of Goiás and Mato Grosso do Sul (Brazil). In this study 624 molluscs of the following species were examined: Drepanotrema anatinum, $D$. lucidum, D. depressissimum, Biomphalaria straminea, D. schrammi, Physa marmorata, Lymnaea columella, Pomacea and Eupera. One to their importance in public health, the discovery of Biomphalaria straminea and Lymnaea columella, intermediate hosts, respectively, of Shistosoma mansoni and Fasciola hepatica, deserves special mention.

Keywords: Mollusca. Disease vectors. Schistosomiasis mansoni. Fascioliasis.

\section{Referências Bibliográficas}

1. CAMARGO, S. Impacto do desenvolvimento na expansão da esquistossomose. Rev. Inst. Med. trop. S.Paulo, 22 (Supl. 4): 117-9, 1980.

2. CUNHA NETO, A.G. Primeiros focos de esquistossomose mansônica em Goiânia, Estado de Goiás, Brasil. Rev. Inst. Med. trop. S. Paulo, 9: 357-8, 1967.

3. DACAL, A.R.C. et al. Susceptibilidade de Lymnaea (Pseudosuccinea) columella (Say, 1817) exposta à infecção por miracídios de Fasciola hepatica (Linnaeus, 1758). Rev. Inst. Med. trop. S.Paulo,30: $361-9,1988$

4. DOUMENGE, J.P. et al. Atlas of the global distribution of schistosomiasis. Geneva, CEGET/CNRS/

WHO, 1987.

5. MAGALHÃES, L.A. Distribuição de moluscos planorbídeos na área do Distrito Federal. Hospital, 76: $725-51,1969$.

6. PARAENSE, W.L. Planorbídeos hospedeiros intermediários do Schistosoma mansoni. In: Cunha, A.S. Esquitossomose mansoni. São Paulo, Sarvier/Ed. USP, 1970. p. 13-30.

7. PARAENSE, W.L. Biomphalaria occidentalis sp.n. from South America (Mollusca, Basommatophora, Pulmonata). Mem. Inst. Oswaldo Cruz, Rio de Janeiro, 76: 199-211, 1981.

8. PARAENSE, W.L. A survey of planorbid mollusks in the Amazonian Region. Mem. Inst. Oswaldo Cruz, Rio de Janeiro, 78: 343-61, 1983.

9. SAIF, M. \& GABER, A. Impacto de desenvolvimento sobre a disseminação da esquitossomose no Egito. Rev. Inst. Med. trop. S. Paulo, 22 (Supl. 4): 20-2, 1980.

Recebido para publicação em 11/5/1990 Aprovado para publicação em 21/08/1990 


\section{Anexo}

Quadro das localidades pesquisadas e espécies de moluscos diagnosticados na área de influência da UHE Couto de Magalhães (maio e julho de 1989).

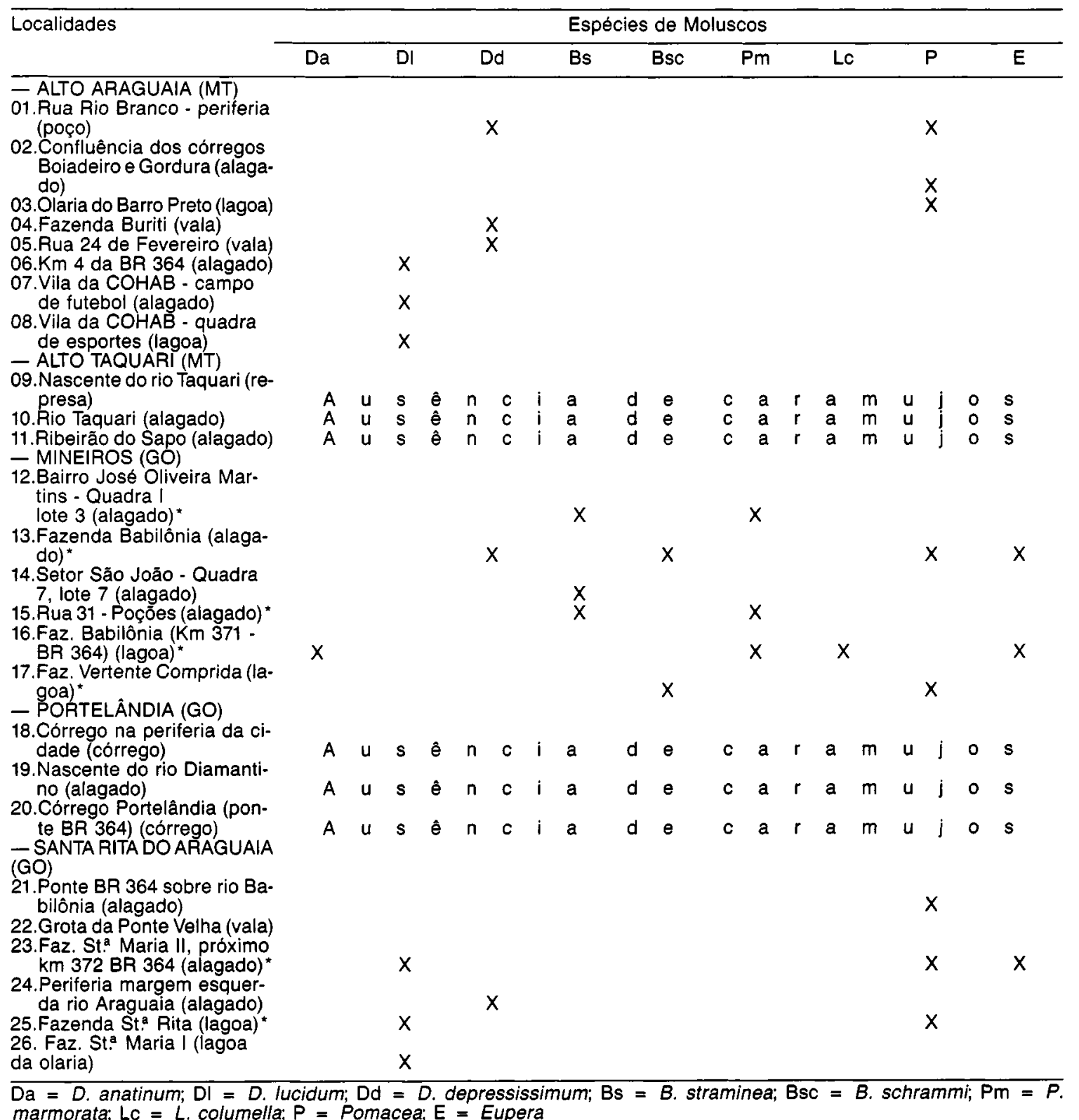

$\left({ }^{*}\right)$ Sintopia 